\title{
CYP1A1m2 polymorphisms regulate estrogen and interleukin-6 in lung cancer
}

\author{
T.T. SREELEKHA ${ }^{1}$, M. RAJESH ${ }^{1}$, V.ANIL KUMAR ${ }^{1}$, J. MADHAVAN ${ }^{2}$ and P. BALARAM ${ }^{3}$ \\ ${ }^{1}$ Division of Cancer Research, Regional Cancer Centre, Trivandrum; ${ }^{2}$ Lung and GI Cancer Division, \\ Regional Cancer Centre, Trivandrum, Kerala, India; ${ }^{3}$ Health Campus, INFORM, \\ Universiti Sains Malaysia, Kubang Kerian, Malaysia
}

Received June 7, 2010; Accepted August 7, 2010

DOI: $10.3892 / \mathrm{mmr} .2010 .352$

\begin{abstract}
Lung cancer, the most common cause of cancerrelated death in men and women, is responsible for 1.3 million deaths worldwide annually. Women are diagnosed to a greater extent than men with adenocarcinoma and small cell carcinoma, both of which are secretory-type tumors. Never smokers diagnosed with lung cancer are also predominantly female, demonstrating the association of genetic factors with lung carcinogenesis. Several epidemiologic studies have associates certain $C Y P 1 A l$ genotypes, alone or in combination, with an increased risk of estrogen-related cancer. The aim of this study was to investigate the impact of the CYP and GST polymorphisms along with estrogen and interleukin-6 (IL-6) levels on the risk of lung cancer. Eighty-six lung cancer patients and 60 controls were included in the study. A significantly higher frequency of polymorphisms in the genes was observed in lung cancer patients compared to controls. Mean estradiol concentration was reduced and IL-6 levels were elevated in patients compared to controls. In conclusion, increased polymorphisms in metabolic genes may be the reason for the reduced estradiol and, thereby, the increased expression of IL-6 in the serum of lung cancer patients.
\end{abstract}

\section{Introduction}

Lung cancer is the leading cause of cancer-related death in both men and women. It occurs most commonly between the ages of 45 and 70, and has become more prevalent in women in the last few decades. In 2005, 90,139 men and 69,078 women in the United States succumbed to lung cancer. That same year, 107,416 men and 89,271 women were diagnosed with lung cancer (1). Recent epidemiologic and biochemical studies suggest a gender difference and increased suscepti-

Correspondence to: $\mathrm{Dr}$ T.T. Sreelekha, Division of Cancer Research, Regional Cancer Centre, Trivandrum, Kerala, India E-mail: lekhasree64@yahoo.co.in

Key words: lung cancer, estrogen, cytochrome P-450, interleukin-6, polymorphism bility to tobacco carcinogens in women compared to men (2). Both endogenous hormones and tobacco may play an important role in carcinogenesis in women. Lung cancer is generally a consequence of chronic exposure over a long period of time to environmental carcinogen mixtures, as well as other environmental, lifestyle, diet and host factors.

It is well known that sex steroids play important roles in various human tissues, including target tissues, as genderdependent factors. Among sex steroids, estrogens are major contributors to cell proliferation (3). Estradiol, the most potent endogenous estrogen, is the important secretory product of the ovary, and represents the principal source of breast cancer causing estrogen in pre-menopausal women. It was reported that estrogen, long known for its role in fueling the growth of breast cancer, may spur the same insidious process in lung cancer (4). Genetic polymorphisms in genes responsible for the metabolism of carcinogens and estrogen underlie individual variations in cancer susceptibility (5). It was reported that genetic polymorphisms in CYP1A1 and GSTM1 are associated with lung cancer risk in Asian populations (6). Possible risk modification by the CYP1A1, GSTM1 and GSTT1 gene polymorphisms of lung cancer susceptibility in a South Indian population was reported from our institute (7). In most reports, the CYP1A1 $\mathrm{m} 1$ and $\mathrm{m} 2$ polymorphisms were shown to be strongly associated with the risk of lung cancer, especially in tobacco users (6-10), whereas the results regarding the association between polymorphisms in GSTM1 and GSTT1 and lung cancer risk have been very inconsistent $(11,12)$.

In addition to metabolic gene polymorphisms, we evaluated the serum concentration of interleukin-6 (IL-6), the production of which is usually inhibited by estrogen. IL-6, a major mediator of the inflammatory response, plays a primary role in the pathophysiology of cancer. Although it has been argued that the cytokine may be secreted by cancer cells, the source of IL-6 in cancer patients has yet to be determined (13). It was reported that estradiol inhibits the production of proliferating cytokines, including IL-6 and macrophage inhibitory factors (14). However, little is known regarding the impact of the CYP and GST polymorphisms along with estrogen and IL-6 levels on the risk of lung cancer. Therefore, the present study evaluated estrogen and IL-6 levels as well as the CYP1A1, GSTM1 and GSTT1 polymorphisms in lung cancer patients and normal controls. 


\section{Materials and methods}

Sample collection. Blood samples from 86 lung cancer patients (37 females, 49 males) were collected from outpatient clinics of the Regional Cancer Centre, Trivandrum. All cases were newly diagnosed and previously untreated. Blood samples from 60 (28 females, 32 males) age- and gender-matched normal controls were also collected by random selection. Serum was separated and stored for analysis of estrogen and IL-6, and DNA was isolated from $3 \mathrm{ml}$ of blood for genomic analysis. At the time of recruitment, each participant was personally interviewed to obtain data on tobacco use. The research protocol was approved by the Institutional Review Board of the Regional Cancer Centre, Trivandrum.

DNA extraction and genotyping. Genomic DNA was isolated by standard procedures (15) from the peripheral blood lymphocytes of lung cancer patients and controls. The CYP1A1m2 genotype was analyzed by a PCR-based restriction fragment length polymorphism (RFLP) method as previously described (16). Null genotypes of GSTM1 and GSTT1 were analysed in a multiplex PCR with the housekeeping gene $\beta$-globin. Primers for the CYP1A1 and GSTs were custom synthesized by Sigma Aldrich (Table I). Appropriate PCR conditions were applied for each primer: an initial denaturing step at $95^{\circ} \mathrm{C}$ for $5 \mathrm{~min}$, followed by 35 cycles of denaturation at $95^{\circ} \mathrm{C}$ for $30 \mathrm{sec}$, annealing at $60-65^{\circ} \mathrm{C}$ for $1 \mathrm{~min}$ and extension at $72^{\circ} \mathrm{C}$ for $1 \mathrm{~min}$, followed by a final extension step at $72^{\circ} \mathrm{C}$ for $5 \mathrm{~min}$. PCR products $(10 \mu \mathrm{l})$ were mixed with $2 \mu \mathrm{l}(6 \mathrm{X})$ loading buffer (bromophenol blue) and loaded onto a $2 \%$ agarose gel along with a 100-bp DNA molecular weight marker, and run at $80 \mathrm{~V}$ for $2 \mathrm{~h}$. Bands at 350, 220, 450 and $110 \mathrm{bp}$ represent the presence of CYP1A1m2, GSTM1, GSTT1 and $\beta$-globin, respectively.

PCR products of CYP1A1m2 were subjected to RFLP analysis. DNA after PCR was precipitated using $100 \%$ ethanol, washed with $70 \%$ ethanol, and dissolved in $10 \mathrm{mM}$ Tris in order to enhance the reaction, since EDTA and excess nucleotides present in the PCR products may reduce enzyme activity. The DNA was then dissolved in $10 \mathrm{mM}$ Tris and incubated with the restriction enzyme $\mathrm{Ncol}$ (IU/ $\mu \mathrm{g}$ DNA) at $37^{\circ} \mathrm{C}$ for $1 \mathrm{~h}$. The digestion products were electrophoresed on $2 \%$ agarose gel, stained with ethidium bromide and analysed using the BioRad Gel Documentation System with Quantity One software. Wild-type alleles generated 232-bp bands, while alleles with the $\mathrm{m} 2$ polymorphism generated 263-bp bands.

Determination of estradiol. ELISA was performed to detect estrogen levels in patient serum as well as in normal serum using the Estradiol kit (Demeditec Diagnostics GmbH), as per the manufacturer's protocol. Briefly, $25 \mu \mathrm{l}$ of calibrators and samples were pipetted into the wells of a 96-well plate. HRP-estradiol conjugate $(200 \mu \mathrm{l})$ was added to each well in sequence followed by incubation for $2 \mathrm{~h}$ at $37^{\circ} \mathrm{C}$ uncovered. The incubation solution was discarded, the wells were rinsed with washing solution three times, and any residual liquid was removed. Immediately thereafter, $100 \mu \mathrm{l}$ of the chromogen/ substrate mixture was pipetted into the wells and incubated for $15 \mathrm{~min}$ at room temperature. The reaction was stopped by
Table I. Oligonucleotide primers used for PCR.

Polymorphic

Primer sequence

gene

CYP1A1m2

F: GAA AGG CTG GGT CCA CCC TCT

R: CCA GGA AGA GAA AGA CCT CCC AGC GGG GCC A

GSTM1 F: GAA CTC CCT GAA AAG CTA AAG C

R: GTT GGG CTC AAA TAT ACG GTG G

GSTT1 F: TTCCTTACTGGTCCTCACATCTC

R: TCACCGGATCATGGCCAGCA

$\beta$-globin $\quad$ F: CAA CTT CAT CCA CGT TCA CC

R: GAA GAG CCA AGG ACA GGT AC

Table II. Characteristics of the study subjects.

\begin{tabular}{lcc}
\hline Variable & Patients $(\mathrm{n}=86)$ & Controls $(\mathrm{n}=60)$ \\
\hline Gender & & 32 \\
Male & 49 & 28 \\
Female & 37 & \\
Age & & $64.49(14.95)$ \\
Mean (SD) & $67.83(12.89)$ & $50-76$ \\
Range & $50-82$ & \\
Smoking status & & 44 \\
Never & 34 & 16 \\
Current & 52 & \\
\hline
\end{tabular}

pipetting $100 \mu \mathrm{l}$ of stop solution into the wells in the same sequence used to dispense the chromogen or substrate mixture. The absorbance of each well was read at $450 \mathrm{~nm}$ within $1 \mathrm{~h}$ of the addition of stop solution.

Determination of IL-6. IL-6 levels were detected in serum samples using a Biolegend Inc. kit according to the manufacturer's protocol. One day prior to running the ELISA, capture antibody was diluted in $1 \mathrm{X}$ coating buffer, and $100 \mu \mathrm{l}$ of the solution was added to each of the 96 wells. The plate was sealed and incubated overnight at $2-8^{\circ} \mathrm{C}$. Plates were washed four times with wash buffer and the residual buffer was blotted by firmly tapping the plates upside down on absorbent paper. To block non-specific binding and reduce the background, $200 \mu \mathrm{l} 1 \mathrm{X}$ assay diluent was added to each well. Sealed plates were incubated at room temperature for $1 \mathrm{~h}$ with shaking. The plate was washed four times with wash buffer, and $100 \mu \mathrm{l}$ of standards and samples were added to the appropriate wells. Incubation and washing was repeated as above, and $100 \mu \mathrm{l}$ of diluted detection antibody was added to each well. The plates were then sealed and incubated at room temperature for $1 \mathrm{~h}$ with shaking and washed with wash buffer four times. Subsequently, $100 \mu \mathrm{l}$ of diluted Av-HRP solution was added to each well followed by incubation at room temperature for 30 min with shaking. The plates were washed five times with 


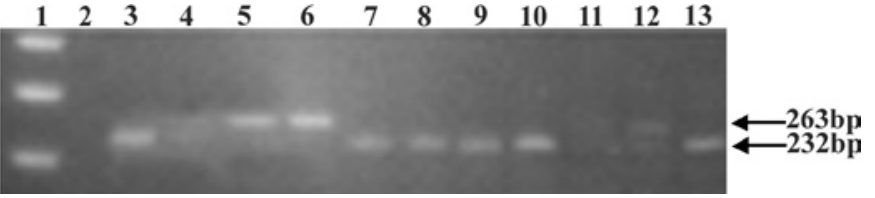

Figure 1. Lane 1, 100-bp molecular weight marker; lane 2, negative control; lanes 3-13, lung cancer patient samples; lanes 4, 11 and 12, showing the heterozygous CYP1A1m2 polymorphism; lanes 5 and 6 , showing the homozygous CYP1A1m2 polymorphism; lanes 3, 7-10 and 13, showing wild-type CYP1A1.

Table III. CYP1A1m2 polymorphism.

\begin{tabular}{lccc}
\hline Subjects & $\begin{array}{c}\text { Heterozygous } \\
\text { polymorphism }\end{array}$ & $\begin{array}{c}\text { Homozygous } \\
\text { polymorphism }\end{array}$ & $\begin{array}{c}\text { Polymorphic } \\
\text { cases (\%) }\end{array}$ \\
\hline $\begin{array}{l}\text { Patients } \\
(\mathrm{n}=86)\end{array}$ & 22 & 14 & $36(41.86)$ \\
$\begin{array}{l}\text { Controls } \\
(\mathrm{n}=60)\end{array}$ & 6 & 4 & $10(16.66)$ \\
\hline
\end{tabular}

Table IV. GSTM1 and GSTT1 polymorphisms.

\begin{tabular}{lcc}
\hline Subjects & $\begin{array}{c}\text { GSTM1 } \\
\text { null deletion }(\%)\end{array}$ & $\begin{array}{c}\text { GSTT1 } \\
\text { null deletion }(\%)\end{array}$ \\
\hline Patients $(\mathrm{n}=86)$ & $23(26.70)$ & $34(39.53)$ \\
Controls $(\mathrm{n}=60)$ & $10(16.66)$ & $4(6.66)$ \\
\hline
\end{tabular}

wash buffer, then $100 \mu 1$ of freshly mixed tetramethylbenzidine substrate was added to each well followed by incubation in the dark for $15 \mathrm{~min}$. Stop solution $(100 \mu \mathrm{l})$ was added to each well and the absorbance was read at $450 \mathrm{~nm}$.

Statistical analysis. Pearson's $\chi^{2}$ test was used to examine differences in the distribution of genetic polymorphisms in the metabolic genes, and in the levels of estrogen and IL-6, between cases and controls. The odds ratio (ORs) with 95\% confidence interval (CI) was calculated using logistic regression.

\section{Results}

The distribution of the study subjects is shown in Table II. There was no significant difference between cases and controls in terms of gender or mean age distribution. Subjects were recruited from the upper age group (>50 years) in order to avoid changes in estrogen levels during the menstrual cycle. The frequency of smoking status differed between the cases and control: more of the patients were smokers as the majority of the male cases were smokers, while the controls were mainly non-smokers. The frequency and duration of the smoking were also different in cases and controls.

Genotyping results showed that the frequency of the CYP1A1m2 polymorphism was $16.66 \%$ (10/60 samples)

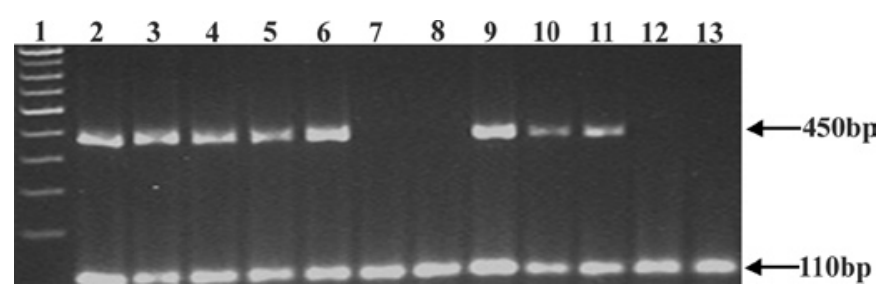

Figure 2. Lane 1, 100-bp molecular weight marker; lanes 2-13, lung cancer patient samples; lanes 7, 8, 12 and 13, showing the GSTT1 deletion; lane 14, negative control.

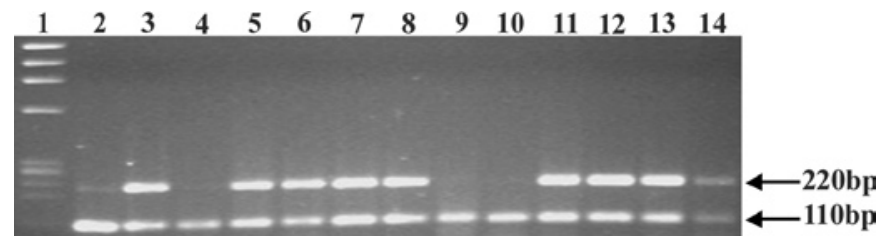

Figure 3. Lane 1, 100-bp molecular weight marker; lanes 2-14, lung cancer patient samples; lanes 2, 4, 9 and 10, showing the GSTM1 deletion.

among the control population, compared to $41.86 \%(36 / 80$ samples) in lung cancer cases (Fig. 1). In the control group, 6 samples (10\%) showed a heterozygous polymorphism (w/m2) and 4 samples $(6.66 \%)$ showed a homozygous polymorphism $(\mathrm{m} 2 / \mathrm{m} 2)$. Out of 36 lung cancer cases, 22 were heterozygous and 14 were homozygous polymorphisms (Table III); this was significantly higher than in the normal controls $(\mathrm{p}<0.000$, $\mathrm{r}=0.347$ ). The frequencies of GSTM1 and GSTT1 deletions were also significantly higher in lung cancer patients compared to controls (Table IV). A GSTT1 null deletion was observed in $40 \%$ of lung cancer patient samples compared to $6.66 \%$ of controls $(\mathrm{p}<0.004, \mathrm{r}=0.364)$. A GSTM1 null deletion was observed in $26.7 \%$ of lung cancer patient samples compared to $10 \%$ of controls $(\mathrm{p}<0.098, \mathrm{r}=0.215)$. In both the GSTM1 and GSTT1 genes, the null deletion was observed less frequently in controls than in lung cancer patients (Figs. 2 and 3).

The mean estradiol concentration in the serum of lung cancer patients was found to be lower in patients (male $29.36 \mathrm{pg} / \mathrm{ml}$, female $28.69 \mathrm{pg} / \mathrm{ml}$ ) than in normal controls (male $59.68 \mathrm{pg} / \mathrm{ml}$, female $91.00 \mathrm{pg} / \mathrm{ml}$ ). Though estradiol concentration varied between males and females, there was a significant difference between patients and normal controls for both genders (Table V). Estradiol concentration was observed to be markedly less in both male and female patients compared to normal controls $(\mathrm{p}<0.000, \mathrm{r}=-0.342)$, whereas IL-6 levels were increased in lung cancer patients (male $218 \mathrm{pg} / \mathrm{ml}$, female $202.16 \mathrm{pg} / \mathrm{ml}$ ) compared to controls (male $117.13 \mathrm{pg} / \mathrm{ml}$, female $101.08 \mathrm{pg} / \mathrm{ml}$ ) (Table V). The CYP1A1m2 polymorphism was found to confer increased risk when associated with decreased serum concentrations of estradiol (OR=7.302; 95\% CI 42.69-74.67). However, the OR increased to 10.28 (95\% CI 139.15-205.97) among individuals with increased levels of serum IL-6 carrying the CYP1A1m2 allele.

The interaction between the CYP1A1 polymorphism and tobacco smoking was also assessed separately for patients and controls. Among smokers, CYP1A1m2 variants conferred an increased risk compared to wild-type genotypes $(p<0.000$, 
Table V. Mean values of estrogen and IL-6 levels in males and females.

\begin{tabular}{|c|c|c|c|c|}
\hline \multirow[t]{2}{*}{ Subjects } & \multicolumn{2}{|c|}{ Mean (SD) of estradiol } & \multicolumn{2}{|c|}{ Mean (SD) of IL-6 } \\
\hline & Male & Female & Male & Female \\
\hline Patients & $29.36(32.96)$ & $28.69 \quad(42.50)$ & $218.09(154.43)$ & $202.16(165.55)$ \\
\hline Controls & $59.68(68.17)$ & $91.00(104.50)$ & 117.13 & $8.08 \quad(98.70)$ \\
\hline
\end{tabular}

$\mathrm{r}=0.394)$. In individuals with the wild-type CYP1A1 genotype who had never smoked, the OR for lung cancer patients for the variant CYP1A1m2 genotype alone and smoking alone was 2.03 (95\% CI 1.24-3.32) and 6.53 (95\% CI 2.43-17.56), respectively.

The risk for lung cancer is increased in patients with metabolic gene polymorphisms and reduced serum estradiol concentrations along with elevated serum IL-6 levels.

\section{Discussion}

Lung cancer is the most common cause of cancer-related death in developed countries. Though tobacco use is believed to be the main cause of lung carcinogenesis, the increase in lung cancer (especially adenocarcinoma) in women who are non-smokers suggests the involvement of factors other than tobacco, such as lifestyle factors. These include increased consumption of fast food and lack of exercise, which are highly associated with cancer. Due to the complex combination of factors behind the development of lung cancer, including carcinogenic exposure, socioeconomic factors, diet and genetics, the specific etiologies of lung cancer are obscure. However, the major causes can be listed as environmental tobacco smoke (17), radon (18), diet $(19,20)$, non-neoplastic lung disease $(21)$ and a family history of lung cancer $(22,23)$.

The present study investigated the association of polymorphisms in metabolic genes involved in the synthesis and metabolism of estradiol (a major component of estrogen that regulates the expression of IL-6 in the body), and their relationship with lung cancer risk in the Indian population.

Estrogen comprises a group of steroid compounds and is produced primarily by developing follicles in the ovaries, the corpus luteum and the placenta. The term 'estrogen' encompasses a group of chemically similar hormones, the most abundant of which are estrone and estradiol. Estrogen directly stimulates the transcription of estrogen-responsive genes in the nucleus of lung cells, and also transactivates growth factor signaling pathways, in particular the epidermal growth factor pathway. Several parameters in lung biology and pathology, both during the developmental stage and in adults, are sexually dimorphic. A role for estrogen in these dimorphisms was suggested in 1980 by Mendelson et al (29), who showed an estrogen-binding component in human fetal lung tissue. In the human population, women are more prone than men to developing chronic obstructive pulmonary disease, and are at a higher risk of developing lung cancer, indicating that women are more susceptible to the deleterious effects of tobacco smoking (30-32). In the present study, we observed that estrogen levels were lower in lung cancer patients than in normal controls, even among female subjects. In order to explain this contradictory result, we evaluated the serum levels of IL- 6 and analyzed polymorphisms of the estrogenmetabolizing gene CYP1A1.

CYP1A1 is a phase I metabolic gene and its mutation is a risk factor for cancer alone and in association with phase II enzymes, such as GSTs.

IL-6 is a major mediator of inflammatory response and plays a primary role in the pathophysiology of cancer. It has been reported that, in addition to hormones, IL-6 also acts as an autocrine growth factor in malignancy. Elevated serum IL-6 levels have been associated with a variety of chronic diseases. IL-6 is produced by macrophages, T cells, B cells, endothelial and tumor cells. IL-6 is capable of promoting tumor growth by up-regulating antiapoptotic and angiogenic proteins in tumor cells, and is associated with poorer survival in patients with metastatic cancers (24). IL-6 receptor engagement leads to the activation of the JAK family of tyrosine kinases, which then stimulate multiple pathways involving MAPKs, PI3Ks, STATs and other signaling proteins (25). In a study of non-small cell lung adenocarcinomas, Gao et al provided additional evidence for the involvement of IL-6 in cancer, and identified an EGFR/ IL-6/STAT3 signaling cascade that plays a key role in tumorigenesis (26). In studies using both mice and human non-small cell lung adenocarcinoma cell lines, Gao et al identified a correlation between activated STAT3 (a downstream target of IL-6) and EGFR mutations in lung tumors. It has also been reported that estrogens suppress IL-6 production in stromal/ osteoblastic cells in vitro.

In this study, it was observed that estradiol levels were lower in the serum of lung cancer patients than in controls. In conjunction with the reduction in estradiol, a significant hike in IL-6 expression was observed. This finding was well supported by the high rate of CYP1A1 polymorphisms, since CYP1A1 is responsible for estrogen metabolism and polymorphisms in CYP1A1 enhance its activity, thereby reducing the estrogen level. Reduced estrogen levels may play a role in the increased serum levels of IL-6.

We observed a significant difference in the distribution of genotype frequencies of polymorphic variants of CYP1A1m2, GSTM1 and GSTT1 among lung cancer patients and normal controls. Our data clearly demonstrate an association between genetic polymorphisms in metabolic genes and increased lung cancer risk. In this study, $41.86 \%$ of the CYP1A1m2 polymorphisms were observed in lung cancer patients, while only $10 \%$ were observed in normal controls. Of these, $61 \%$ were heterozygous polymorphisms and 39\% homozygous polymorphisms, as evidenced by the obtained bands. In most of the patients, heterozygous polymorphisms were predomi- 
nant. It was observed that polymorphisms were mostly expressed in female lung cancer patients. A high-risk group for lung carcinoma has been previously reported in terms of polymorphisms in the CYP1A1, GSTM1 and GSTT1 genes $(6,7)$. Several other studies have reported an effect of the CYP and GST polymorphisms on lung cancer risk (33). We studied the significance of genetic polymorphisms in the CYP1A1, GSTM1 and GSTT1 genes in patients with oral cancer in association with tobacco smoke (16). An increased null deletion in the GSTM1 and GSTT1 genes was observed in oral cancer patients compared to controls. In the case of CYP1A1, a significantly higher frequency of polymorphisms was evident in the $\mathrm{m} 2$ allele in oral cancer patients. Since null genotype individuals may be poor detoxifiers with a reduced ability to neutralize reactive carcinogenic intermediates, they are potentially a high-risk category. The frequency distribution of the CYP1A1 m2 (Il/val) genotype among oral cancer patients was significantly different from that of normal controls. The risk of CYP1A1 is supported by the functional difference between the presence of valine and isoleucine; valine type has higher catalytic and mutagenic activity towards benzo[a]pyrene than isoleucine type. However, no studies have reported on the role of estrogen in the risk of lung cancer alone or in association with metabolic gene polymorphisms.

An increasing incidence of lung cancer in women has recently been reported from our clinics. It has also been noted that the incidence of lung cancer in women is associated with an urban population. In India, urban-dwelling women generally work outside the home and must travel mainly by public conveyances. Vehicular exhaust is a major risk factor for lung carcinogenesis. Polycyclic aromatic hydrocarbons (PAHs) are a group of chemicals that are formed during the incomplete combustion of diesel, tobacco, oil and gas, and other organic substances. Ambient air contaminated with PAHs is highly associated with lung carcinogenesis. It was reported that Chinese women, many of whom are non-smokers, have very high lung cancer rates. This phenomenon has been associated with exposure to cooking oil vapours and other forms of air pollution in the indoor environments of China (27). A gene-environment interaction has been well demonstrated previously, and may stimulate the onset of gene mutations. Environmental carcinogens may be activated enzymatically to chemically reactive electrophiles that form carcinogen DNA adducts. It has been hypothesized that an individual's susceptibility to cancer may be partially affected by the balance between the capacity to activate inhaled pro-carcinogens (Phase I enzymes) and the capacity to detoxify carcinogens (Phase II enzymes) (28). It is increasingly recognized that genetic polymorphisms common in the population can affect each of these processes.

In conclusion, reduced expression of estrogen in lung cancer patients was observed, which was verified by the increased expression of IL-6 and the high frequency of CYP1A1 polymorphisms. Estrogen is believed to be a fueling agent in the development of various types of cancer, in particular breast cancer. However, its role in lung cancer has not been explained in detail. In the present study, the observed reduction in estrogen levels in the serum of lung cancer patients is a novel finding, and was supported by the high rate of CYP1A1 polymorphisms and the increased expression of IL-6 in the serum of lung cancer patients. Completing the analysis using a large number of samples may help to develop serum estrogen content as a biomarker for lung carcinogenesis.

\section{Acknowledgements}

This study was supported by the Department of Science and Technology, Government of India (grant no. SR/SO/ HS-107/2005).

\section{References}

1. US Cancer Statistics Working Group. United States Cancer Statistics: 1999-2005 Incidence and Mortality Web-based Report. Atlanta, GA: Department of Health and Human Services, Centers for Disease Control and Prevention; Washington, DC: National Cancer Institute; 2009. www.cdc.gov/uscs.

2. Sin DD, Cohen SB, Day A, Coxson H and Paré PD: Understanding the biological differences in susceptibility to chronic obstructive pulmonary disease between men and women. Proc Am Thorac Soc 4: 671-674, 2007.

3. Niikawa H, Suzuki T, MiKi Y, et al: Intratumoral estrogens and estrogen receptors in human non-small cell lung carcinoma. Clin Cancer Res 14: 4417-4426, 2008.

4. Melville K: Estrogen May Fuel Lung Cancer Growth. April 2000. http://www.scienceagogo.com/news/20000302190630data_ trunc_sys.shtml.

5. Tsuchiya Y and Nakajima MT: Cytochrome P450-mediated metabolism of estrogens and its regulation in human. Cancer Lett 227: 115-124, 2005.

6. Lee KM, Kang D, Clapper ML, et al: CYP1A1, GSTM1, and GSTT1 polymorphisms, smoking, and lung cancer risk in a pooled analysis among Asian populations. Cancer Epidemiol Biomarkers Prev 17: 1120-1126, 2008

7. Sreeja L, Syamala V, Hariharan S, Madhavan J, Sivanandan CD and Ankathil R: Possible risk modification by CYP1A1, GSTM1 and GSTT1 gene polymorphisms in lung cancer susceptibility in a South Indian population. J Hum Genet 50: 618-627, 2005.

8. Shaffi SM, Shah MA, Bhat IA, Koul P, Ahmad SN and Siddiqi MA: CYP1A1 polymorphisms and risk of lung cancer in the ethnic Kashmiri population. Asian Pacific J Cancer Prev 10: 651-656, 2009.

9. Wright CM, Larsen JE, Colosimo ML, et al: Genetic association study of CYP1A1 polymorphisms identifies risk haplotypes in nonsmall cell lung cancer. Eur Respir J 35: 152-159, 2010.

10. Pisani P, Srivatanakul P, Anderson-Moor JR, et al: GSTM1 and CYP1A1 polymorphisms, tobacco, air pollution and lung cancer: a study in rural Thailand. Cancer Epidemiol Biomarkers Prev 15: 667-674, 2006.

11. Schneider J, Bernges U, Philipp M and Woitowitz HJ: GSTM1, GSTT1, and GSTP1 polymorphism and lung cancer risk in relation to tobacco smoking. Cancer Lett 208: 65-74, 2004.

12. Zhuo X, Cai L, Xiang Z, Li Q and Zhang X: GSTM1 and GSTT1 polymorphisms and nasopharyngeal cancer risk: an evidencebased meta-analysis. J Exp Clin Cancer Res 28: 461-468, 2009.

13. Hodge DR, Hurt EM and Farrar WL: The role of IL-6 and STAT3 in inflammation and cancer. Eur J Cancer 41: 2502-2512, 2005.

14. Pottratz ST, Bellido T, Mocharla H, Crabb D and Manolagas SC: 17 beta-estradiol inhibits expression of human interleukin-6 promoter receptor constructs by a receptor dependent mechanism. J Clin Invest 93: 944-950, 1994.

15. Sambrook J, Fritsch EF and Maniatis T: Molecular Cloning, A Laboratory Manual. 2nd edition. Cold Spring Harbor Laboratory, Cold Spring Harbor, NY, 1989.

16. Sreelekha TT, Ramadas K, Pandey M, Thomas G, Nalinakumari KR and Pillai MR: Genetic polymorphism of CYP1 A1, GST M1 and GSTT1 genes in Indian oral cancer. Oral Oncol 37: 593-598, 2001.

17. Brownson RC, Alavanja MC, Hock ET and Loy TS: Passive smoking and lung cancer in nonsmoking women. Am J Public Health 82: 1525-1530, 1992.

18. Samet JM, Brenner D, Brooks AL, et al: Health effects of exposure to radon: BEIR VI. National Academy Press, Washington (DC), 1998. 
19. Alavanja MC, Brownson RC and Benichou J: Estimating the effect of dietary fat on the risk of lung cancer in nonsmoking women. Lung Cancer 14 (Suppl 1): 63-74, 1996.

20. Alavanja MC, Brown CC, Swanson C and Brownson RC: Saturated fat intake and lung cancer risk among nonsmoking women in Missouri. J Natl Cancer Inst 85: 1906-1916, 1993.

21. Wu AH, Fontham ET, Reynolds P, et al: Previous lung disease and risk of lung cancer among lifetime nonsmoking women in the United States. Am J Epidemiol 141: 1023-1032, 1995.

22. Alavanja MC, Brownson RC, Benichou J, Swanson C and Boice JD Jr: Attributable risk of lung cancer in lifetime nonsmokers and long-term ex-smokers (Missouri, United States). Cancer Causes Control 6: 209-216, 1995.

23. Sellers TA, Potter JD, Bailey-Wilson JE, Rich SS, Rothschild H and Elston RC: Lung cancer detection and prevention: evidence for an interaction between smoking and genetic predisposition. Cancer Res 52 (Suppl 9): 2694-2697, 1992.

24. Mendelson CR, MacDonald PC and Johnston JM: Estrogen binding in human foetal lung tissue cytosol. Endocrinology 106 : 368-374, 1980 .

25. Prescott E, Bjerg AM, Andersen P, Lange and Vestbo J: Gender difference in smoking effects on lung function and risk of hospitalization for COPD: results from a Danish longitudinal population study. Eur Respir J 10: 822-827, 1997.

26. Kmietowicz Z: Women at double risk of small cell lung cancer. BMJ 317: 1614, 1998
27. Zang EA and Wynder EL: Differences in lung cancer risk between men and women: examination of the evidence. J Natl Cancer Inst 88: 183-192, 1996.

28. Salgado R, Junius S, Benoy I, et al: Circulating interleukin-6 predicts survival in patients with metastatic breast cancer. Int $J$ Cancer 103: 642-646, 2003.

29. Hong DS, Angelo LS and Kurzrock R: Interleukin-6 and its receptor in cancer: implications for translational therapeutics. Cancer 110: 1911-1928, 2007.

30. Gao SP, Mark KG, Leslie K, et al: Mutations in the EGFR kinase domain mediate STAT3 activation via IL-6 production in human lung adenocarcinomas. J Clin Invest 117: 3846-3856, 2007.

31. Metayer C: Cooking oil fumes and risk of lung cancer in women in rural Gansu, China. Lung Cancer 35: 111-117, 2002.

32. Gaspar P, Moreira J, Kvitko K, Torres M, Moreira A and Weimer T: CYP1A1, CYP2E1, GSTM1, GSTT1, GSTP1, and TP53 polymorphisms: do they indicate susceptibility to chronic obstructive pulmonary disease and non-small-cell lung cancer? Genet Mol Biol 27: doi: 10.1590/S1415-47572004000200001. 2004.

33. Spivack SD, Fasco MJ, Walker VE and Kaminsky LS: The molecular epidemiology of lung cancer. Crit Rev Toxicol 27: 319-365, 1997. 